\title{
Characterizing demographics, comorbidities, and costs of care among populations with Duchenne muscular dystrophy with Medicaid and commercial coverage
}

\author{
Alexa C Klimchak, MA; Shelagh M Szabo, MSc; Christina Qian, MSc; Evan Popoff, MSc; Susan Iannaccone, MD; \\ and Katherine L Gooch, PhD
}

\section{What is already known about this subject}

- Duchenne muscular dystrophy (DMD) is a severe progressive neuromuscular degenerative disease; afflicted patients require extensive management over their lifespan.

- Claims-based studies of outcomes among those with DMD are very few; as a result, little is known about outcomes among patients with DMD covered by different payer types.

\section{ABSTRACT}

BACKGROUND: Duchenne muscular dystrophy (DMD) is a severe $X$-linked progressive neurodegenerative disease characterized by loss of ambulation, cardiomyopathy, respiratory insufficiency, and early mortality. Few data are available that describe the direct medical costs among patients with DMD in the United States.

OBJECTIVE: To characterize the demographics, comorbidity burden, and direct monthly costs of care among patients with DMD with

\author{
What this study adds \\ - This large retrospective observational \\ study evaluated the characteristics of, \\ and the direct medical costs of care, \\ among almost 2,000 patients covered \\ under commercial or Medicaid plans in \\ the United States. \\ - In this study, the distribution of \\ demographic characteristics was \\ comparable between DMD patients \\ with commercial coverage and those \\ with Medicaid coverage, and monthly \\ direct medical costs were similar, \\ providing contemporary data on the \\ characteristics of DMD patients and \\ their attendant economic burden.
}

\section{Author affiliations}

Alexa C Klimchak, MA, and Katherine L Gooch, PhD, Sarepta Therapeutics, Inc., Cambridge, MA. Shelagh M Szabo, MSc; Christina Qian, MSc; and Evan Popoff, MSc, Broadstreet HEOR, Vancouver BC Canada. Susan Iannaccone, MD, Children's Medical Center Ambulatory Care Pavilion, University of Texas Southwestern Medical Center, Dallas.

\section{AUTHOR CORRESPONDENCE:}

Shelagh M Szabo, 604.800.0648;

sszabo@broadstreetheor.com

J Manag Care Spec Pharm 2021;27(10):1426-37

Copyright $\odot 2021$, Academy of Managed Care Pharmacy. All rights reserved.
Medicaid and with commercial insurance coverage.

METHODS: IBM MarketScan Commercial and Multi-State Medicaid claims (2013-2018) were used to identify males aged 30 years or under with diagnostic codes for muscular dystrophy or DMD; additional exclusion criteria were applied to identify those with probable DMD. Baseline characteristics and comorbidities were tabulated. The frequency of health care resource use and median (interquartile range [IQR]) monthly costs (in 2018 USD) were estimated from those with at least 12 months of continuous follow-up.
RESULTS: Median (IQR) baseline ages were similar between the Medicaid (14 [9-20] years; $n=2,007)$ and commercial (15 [9-21] years; $n=1,964$ ) DMD cohorts. The frequency of comorbidities over the period was slightly higher with those on Medicaid. The median duration of follow-up was 3.1 years among members of the Medicaid DMD cohort and 1.7 years among the commercial DMD cohort. Median monthly resource use was generally higher among the Medicaid DMD cohort; nonetheless, median (IQR) monthly costs were similar at $\$ 1,735(\$ 367-\$ 5,281)$ for the Medicaid DMD cohort vs $\$ 1,883$ ( $\$ 657$ $\$ 6,796)$ for the commercial DMD cohort. 
CONCLUSIONS: The demographic characteristics and median direct medical costs were similar between patients with commercial vs Medicaid coverage, even though patients with Medicaid coverage had higher resource use. Despite challenges in definitively identifying DMD patients using claims data, these findings help characterize contemporary DMD populations in the United States and the related direct economic burden to the payer.

Duchenne muscular dystrophy (DMD) is a rare, X-linked neuromuscular disorder caused by mutations in the gene for dystrophin., ${ }^{1,2}$ Patients typically present in early childhood with gait abnormalities, muscle weakness, and delayed motor and cognitive function., ${ }^{1,3-6}$ Data from neuromuscular patient registries, including the Cooperative International Neuromuscular Research Group, ${ }^{7}$ Duchenne Registry, ${ }^{8}$ and Centers for Disease Control and Prevention's Muscular Dystrophy Surveillance, Tracking, and Research Network have documented the relentless progression of DMD. ${ }^{9}$ When left untreated, progressive muscular degeneration leads to loss of ambulation often before age 12 years, cardiomyopathy and respiratory insufficiency in late childhood or early adulthood, and early mortality from the late teens into the third decade of life. ${ }^{3,7,10}$

Rare, severe conditions such as DMD are associated with considerable patient burden and substantial economic impact, ${ }^{11,12}$ due to the progression of the disease and the accompanying effects of the constellation of associated comorbidities. ${ }^{12-21}$ Despite rigorous evidence of the clinical burden of DMD progression, and while it is clear that patients with DMD require intensive follow-up and clinical care, $, 11,20-23$ little is known about outcomes among patients with DMD managed under different care plans in the United States. While claims database studies are often used to characterize patient populations, understand treatment patterns, and assess aspects of the economic burden of a health condition, such assessments for DMD are limited. The only claims-based study providing such estimates was before the widespread use of corticosteroids and included a small number of patients from 1 commercial plan only. ${ }^{22}$

Analyses of outcomes among those treated under different payer segments are lacking, specifically among individuals accessing care through commercial plans (eg, private or employer-sponsored health insurance) or government-sponsored health programs (eg, Medicaid). Broader assessments of patients with different payer types are critical, since how treatment is funded would be an important contributor to the economic burden of DMD. Such data, as well as contemporary information on the characteristics and duration of DMD patients within commercial and Medicaid plans over time, is imperative to understanding the unmet need and patient burden in DMD.

The objective of this study was to characterize DMD populations in the United States with commercial and Medicaid insurance coverage, including their demographics, comorbidity burden, and direct monthly costs of care.

\section{Methods}

This study was a US-based, real-world, retrospective cohort study that described the clinical and economic burden among those with DMD.

\section{DATA SOURCE}

Data were derived from the IBM MarketScan commercial and Multi-State Medicaid databases, ${ }^{24,25}$ each of which are large, nationally representative health care databases. The 2 datasets are distinct and do not have a mechanism to identify patients who are present in the datasets simultaneously (ie, they have dual coverage) or consecutively (ie, they changed insurance coverage). These data have been widely validated for clinical, pharmacoepidemiological, and pharmacoeconomic research ${ }^{26-29}$ and allow for robust estimation of treatment patterns, health care resource use, and costs. ${ }^{30-34}$

\section{STUDY SAMPLE}

The eligible DMD population included all males aged 30 years and under, with a hereditary progressive muscular dystrophy (MD) International Classification of Diseases, Ninth Revision, Clinical Modification (ICD-9-CM) diagnostic code 359.1 until September 30, 2015; MD International Classification of Diseases, Tenth Revision, Clinical Modification (ICD-10-CM) code G71.0 from October 1, 2015; the Becker/Duchenne MD-specific ICD-10-CM diagnostic code G71.01 from October 1, 2018, in any position on at least 2 outpatient DMD medical claims (with $>30$ days between claims) or as the primary or secondary diagnosis of at least 1 inpatient claim; or a dispensation for eteplirsen (specific to exon 51 skip amenable patients only).

To remove those likely to have other congenital dystrophies (where loss of function would have occurred earlier) and focus on those with "probable DMD," individuals with the following codes were excluded (see Supplementary Table 1 for applicable codes, available in online article) $)^{22}$ : at least 2 medical claims for ventilator use at least 180 days apart, before age 6 years; at least 1 medical claim with a Current Procedural Terminology code for an orthopedic procedure on the foot before age 3 years; at least 1 medical claim for a power, power-assist, and/or manual wheelchair 
before age 5 years; or at least 1 medication fill (National Drug Code number 64406005801) or an injection code (Healthcare Common Procedure Coding System J2326) for nusinersen at any point during the study period.

No exclusion criteria related to the duration of follow-up were imposed, except when noted for resource use and direct costs analysis. Commercially insured DMD patients comprised the "commercial DMD cohort," and Medicaidinsured DMD patients made up the "Medicaid DMD cohort."

An unaffected comparison group of registrants of each insurance type was also included, with whom to compare estimates of the duration of available follow-up, rates of comorbidities, and economic burden. The comparison group was drawn from all of those without a diagnostic code for MD over the study period and matched to the DMD group on age at index date, sex, and calendar month of index date. Separate comparison groups were identified for both the commercial and Medicaid DMD cohorts.

\section{STUDY DESIGN}

Because of differences in data availability, the identification period for study enrollment was April 1, 2013, to March 31,2018 , for those with commercial coverage and January 1, 2013, to June 30, 2018, for those with Medicaid coverage. All DMD cohort members were indexed via the first eligible inpatient or outpatient visit with an MD code. All individuals were followed until death (if known), deregistration, or the end of the follow-up period.

\section{OUTCOMES AND ANALYSIS}

To characterize the population with and without DMD in the commercial and Medicaid cohorts, the median age, distribution of age, and geographic region at baseline were described. Median follow-up time was estimated per cohort. Health status of the cohorts over the study period was described by the frequency and characteristics of corticosteroid use, an unweighted Elixhauser Comorbidity Index score ${ }^{35}$ and the frequency of key neurologic/neuropsychiatric and other comorbidities (identified from a targeted literature review and clinical expert consultation, as described in Supplementary Table 2, available in online article).

Median follow-up time and attrition, defined by individuals exiting coverage in their respective payer insurance type for any reason, was estimated over time using KaplanMeier analysis based on individual time since cohort entry. The reason for attrition (eg, due to mortality vs switching insurance plans) was not available in the datasets.

All-cause resource use and direct medical costs were summarized among the subgroup that had at least 12 months of continuous follow-up after index. Costs and resource use because of outpatient visits, hospitalizations, and medication use were considered; costs for emergency room visits are accounted for in the MarketScan datasets within inpatient and outpatient costs. Total gross payment to all providers (total eligible charges less any reasonable and customary amounts and discounts for preferred provider organization services, but before reductions for deductibles, copayments, and other savings) were used to estimate costs. Resource use was summarized by median (with interquartile range [IQR]), as well as the percentage using the resource. Median monthly costs were calculated, and all costs were inflated to 2018 US dollars (USD). ${ }^{36}$

The impact of key design assumptions on the results were tested in sensitivity analyses. To increase the likelihood of eliminating uncertain DMD cases, the definition of DMD was varied using 2 additional cohort definitions: (1) adding an inclusion criterion of having at least 1 diagnosis from a specialist and (2) adding an exclusion criterion of having at least 2 claims with an ICD-9-CM or ICD-10-CM diagnostic code for any of the following: myoneural disorder (358.x or G70.x), Guillain-Barre syndrome (357.0 or G61.0), or hereditary motor and sensory neuropathy (356.2 or G60.0).

Results derived from the DMD cohorts identified by the sensitivity analysis definitions were compared with those from the base-case DMD cohorts using the median (IQR) age at index, Elixhauser Comorbidity Index score, and monthly costs. Two additional sensitivity analyses were performed: (1) to reduce potential bias due to the impact of varying follow-up time, resource use was estimated from the subgroup with at least 12 months of continuous follow-up after index for the first year of follow-up only and (2) missing costs data for all encounters where a \$0 charge was assigned were imputed by assigning the average cost per resource type observed, and median monthly costs were calculated and statistically compared using the MannWhitney U Test. Statistical analyses were conducted using $\mathrm{R}$ version 4.0.4. (R Foundation for Statistical Computing).

\section{Results}

\section{PATIENT CHARACTERISTICS}

Over the study period, 1,964 individuals with DMD were identified from the commercial dataset and 2,007 from Medicaid (Figure 1). The age distributions (Supplementary Figure 1, available in online article) and median age at baseline were similar between the DMD cohorts (Table 1). By design, the age distribution was similar for the DMD cohorts and their respective comparison cohorts. The median (IQR) duration of follow-up was longer among members of the 


\section{FIGURE 1 Study Design and Enrollment by Cohort}

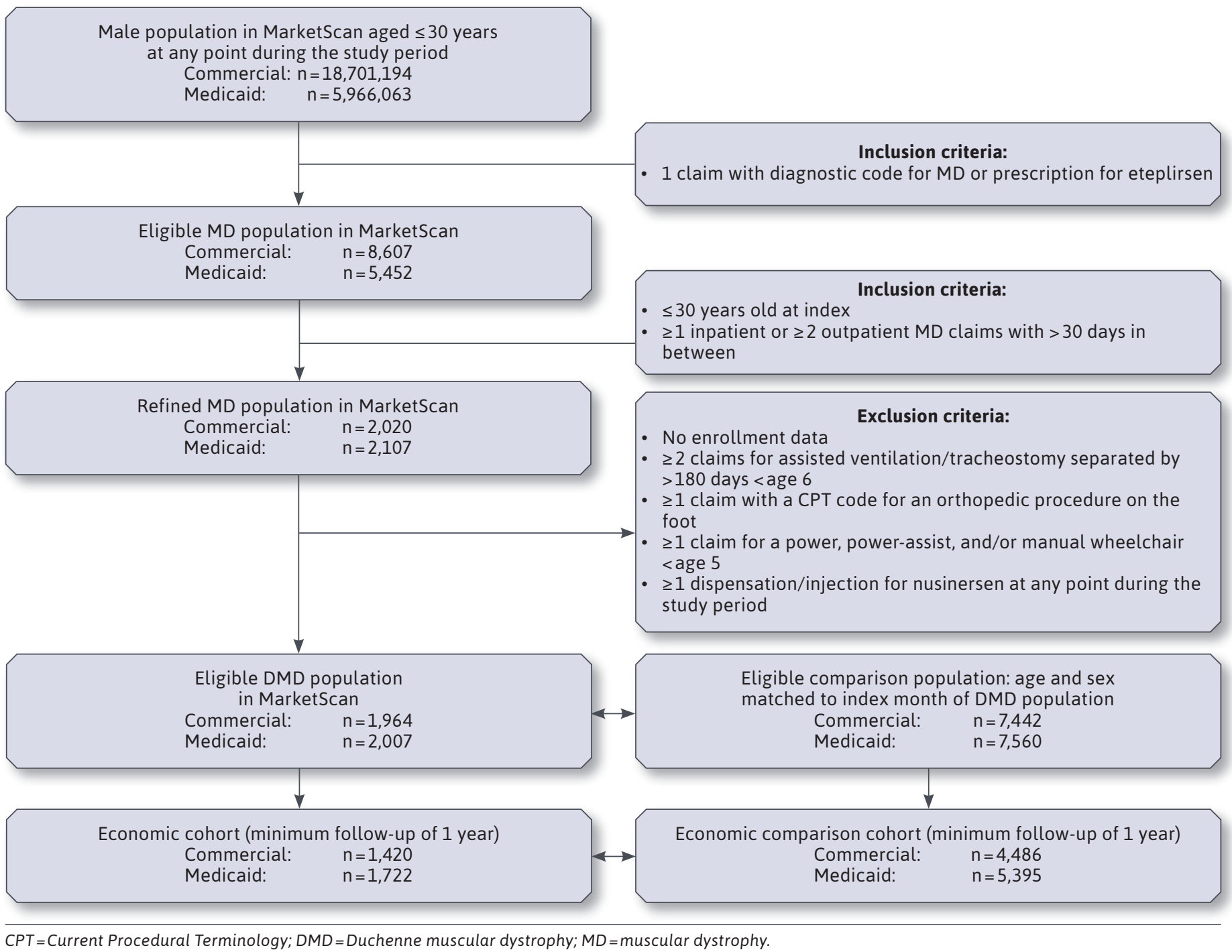

Medicaid cohort (3.1 [1.6-4.7] years) compared with the commercial DMD cohort (1.7 [0.9-3.4] years), with $68.9 \%$ of the Medicaid DMD cohort (vs $47.4 \%$ of the commercial DMD cohort) remaining in the cohorts at 3 years when accounting for censoring. The percentage of the non-DMD comparison cohorts remaining at 3 years was also higher among the Medicaid cohort (57.7\%) than the commercial (37.2\%) cohort.

Less than half of patients were observed to receive corticosteroid treatment (and fewer in the commercial DMD cohort [38.8\%] than in Medicaid [46.1\%]; Table 1) over their available follow-up. Of those who received corticosteroids, the majority received prednisone/prednisolone, and a minority received deflazacort $(17.2 \%$ of the commercial DMD cohort on any corticosteroid and $8.3 \%$ of the Medicaid DMD cohort). Thirty patients in the commercial DMD cohort (1.5\%) and $16(0.8 \%)$ in the Medicaid DMD cohort were treated with eteplirsen.

The comorbidity burden, by median (IQR) Elixhauser scale score, was lower among the commercial (1 [0-3]) compared with the Medicaid (2 [1-4]) DMD cohorts. A lower percentage of patients in the commercial DMD cohort 


\section{TABLE 1 Baseline Demographics, Health Status and Comorbidities Over Study Period, Commercial and Medicaid DMD, and Comparison Cohorts}

\begin{tabular}{|c|c|c|c|c|}
\hline & \multicolumn{2}{|c|}{ Commercial } & \multicolumn{2}{|c|}{ Medicaid } \\
\hline & DMD & Comparison & DMD & Comparison \\
\hline & $(n=1,964)$ & $(n=7,442)$ & $(n=2,007)$ & $(n=7,560)$ \\
\hline Follow-up, years, median (IQR) ${ }^{\mathrm{a}}$ & $1.7(0.9-3.4)$ & $1.4(0.6-3.0)$ & $3.1(1.6-4.7)$ & $2.0(0.8-3.8)$ \\
\hline Age at cohort entry, median (IQR) & $15 \quad(9-21)$ & $15 \quad(9-20)$ & $14 \quad(9-20)$ & $(9-19)$ \\
\hline \multicolumn{5}{|l|}{ Patients, by age category, $\mathrm{n}(\%)$} \\
\hline $0-3$ & $(4.5)$ & (4.3) & $(5.7)$ & $(5.2)$ \\
\hline $4-7$ & $260 \quad(13.2)$ & $1,005 \quad(13.5)$ & $281 \quad(14.0)$ & 1,054 \\
\hline $8-13$ & $497 \quad(25.3)$ & 1,922 & $(26.6)$ & 2,061 \\
\hline $14-17$ & $379 \quad(19.3)$ & $1,478 \quad(19.9)$ & $361 \quad(18.0)$ & 1,427 \\
\hline $18-25$ & $527 \quad(26.8)$ & 2,012 & $(26.8)$ & 1,988 \\
\hline $26+$ & $213 \quad(10.8)$ & (9.5) & $(9.0)$ & $(8.5)$ \\
\hline \multicolumn{5}{|l|}{ Patients, by region, ${ }^{\mathrm{b}} \mathrm{n}(\%)$} \\
\hline Northeast & $355 \quad(18.1)$ & 1,332 & NA & NA \\
\hline North central & $515 \quad(26.2)$ & $1,926 \quad(25.9)$ & NA & NA \\
\hline South & $735 \quad(37.4)$ & $2,756 \quad(37.0)$ & NA & NA \\
\hline West & $358 \quad(18.2)$ & $1,367 \quad(18.4)$ & NA & NA \\
\hline Unknown & - & $(0.8)$ & NA & NA \\
\hline \multicolumn{5}{|l|}{ Patients with CS use, by CS type, ${ }^{\mathrm{b}} \mathrm{n}(\%)$} \\
\hline Any CS use & $762 \quad(38.8)$ & $(13.3)$ & $(46.1)$ & $(17.8)$ \\
\hline Prednisone/prednisolone & $694 \quad(35.3)$ & $990 \quad(13.3)$ & $896 \quad(44.6)$ & $1343 \quad(17.8)$ \\
\hline Deflazacort & $(6.7)$ & - & (3.8) & - \\
\hline None & $1,202 \quad(61.2)$ & 6,452 & 1,081 & 6,217 \\
\hline Age at first CS use, ${ }^{a}$ median (IQR) & $14 \quad(9-19)$ & $16 \quad(11-21)$ & $(8-18)$ & $(9-19)$ \\
\hline PDC among CS users & $0.38(0.04-0.95)$ & $0.02(0.01-0.04)$ & $0.12(0.01-0.56)$ & $0.01(0.01-0.02)$ \\
\hline
\end{tabular}

was observed to have neurological and neuropsychiatric comorbidities (36.3\% vs 58.3\%) and non-neurological and non-neuropsychiatric comorbidities (58.7\% vs 66.8\%), compared with the Medicaid DMD cohort.

\section{HEALTH CARE RESOURCE USE}

The subset of patients with at least 1 year of continuous follow-up was identified from each of the commercial $(n=1,420$; $72.3 \%)$ and Medicaid $(\mathrm{n}=1,722 ; 85.8 \%)$ DMD study cohorts. While median (IQR) follow-up was longer for the DMD economic cohort subsets (2.7 [1.7-4.0] years for the commercial DMD; 3.6 [2.1-4.7] years for the Medicaid DMD cohorts), demographic characteristics were similar to the main DMD cohorts. Among the DMD patients included in the economic analyses, the median (IQR) ages at cohort entry were also 15
(9-21; commercial) and 14 (9-20; Medicaid) years; the median (IQR) Elixhauser score was 2 (1-3; commercial) and 2 (1-4; Medicaid; Supplementary Table 3 , available in online article).

Median monthly resource use was generally higher among the Medicaid DMD cohort than the commercial DMD cohort (Table 2). A median of 3.8 outpatient visits per patient per month was observed among the Medicaid DMD cohort vs 1.4 among the commercial DMD cohort. Inpatient visits were less frequent, with $31.1 \%$ of Medicaid and $25.9 \%$ of commercial patients having at least 1 hospitalization during their follow-up; the median length of stay was 4.3 (Medicaid) and 4.0 (commercial) days. More Medicaid DMD patients had at least 1 emergency department (ED) visit during their follow-up (64.6\% of Medicaid DMD patients vs $47.8 \%$ of commercial DMD patients). Among the DMD 


\section{TABLE 1 Baseline Demographics, Health Status and Comorbidities Over Study Period, Commercial and Medicaid DMD, and Comparison Cohorts (continued)}

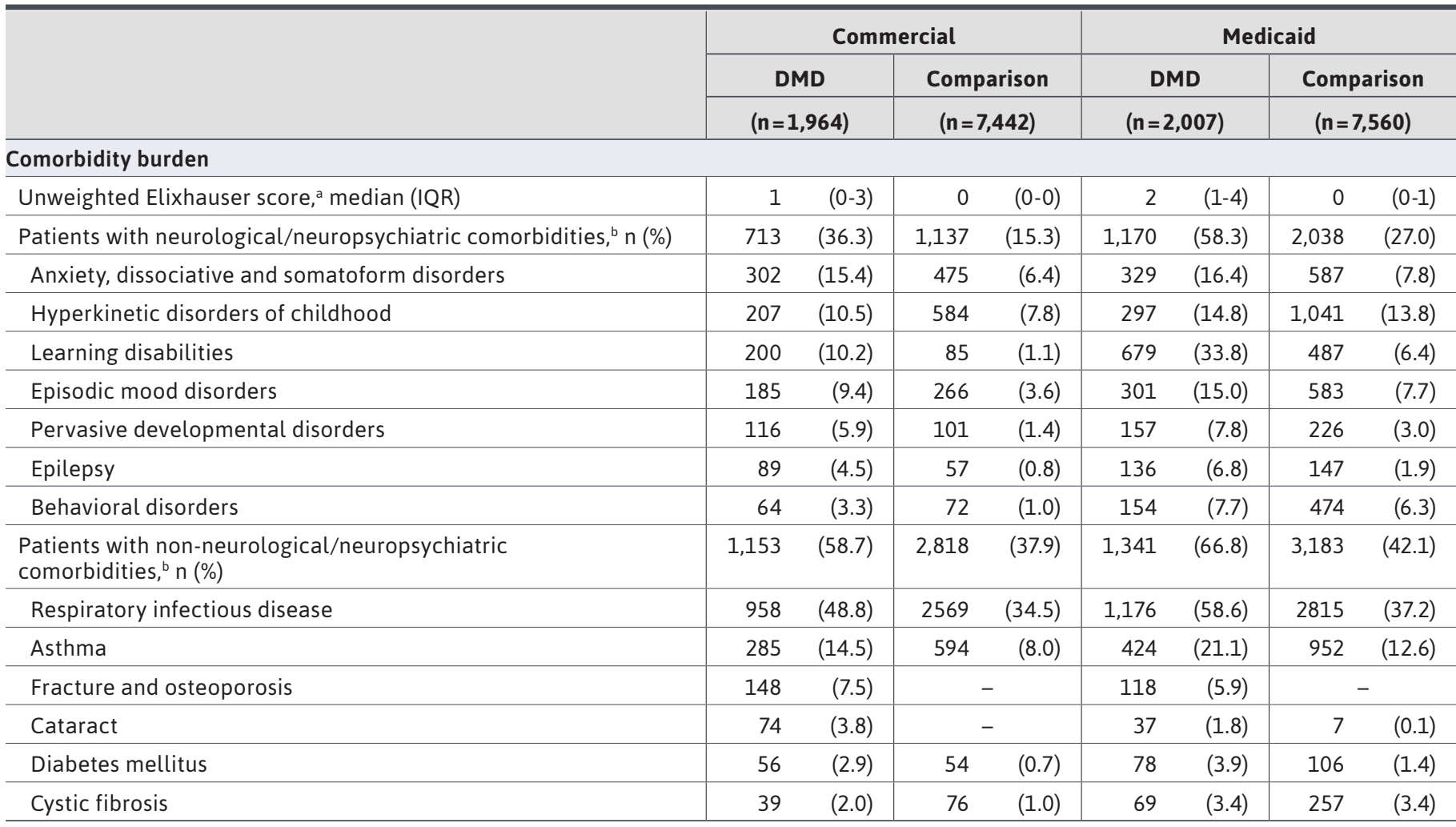

Note: Dash represents $n<5$.

aStatistical significance estimated using Mann-Whitney $U$ test.

${ }^{b}$ Statistical significance estimated using 2-sample Student's t-test.

$C S=$ corticosteroid $; D M D=$ Duchenne muscular dystrophy; $I Q R=$ interquartile range; $N A=$ not available; $P D C=$ proportion of days covered.

cohorts, $68.5 \%$ of Medicaid patients and $64.5 \%$ of commercial patients had at least 1 general practitioner visit over the follow-up period, and almost all (97.0\%) of the commercial DMD cohort visited a specialist during their follow-up (compared with $75.1 \%$ of the Medicaid DMD cohort).

Resource use was substantially higher for the DMD cohorts than for the comparison non-DMD cohorts. A greater proportion of patients in the DMD cohorts had hospitalizations, ED visits, and outpatient visits, along with higher medications use, than in the non-DMD comparison cohorts (Table 2).

\section{DIRECT MEDICAL COSTS OF CARE}

While average resource use tended to be lower among members of the commercial DMD cohort, average total monthly costs were higher than in the Medicaid DMD cohort (Figure 2 and Supplementary Table 4, available in online article). Median (IQR) monthly costs were $\$ 1,883$ (\$657-\$6,796) for the commercial DMD cohort vs. \$1,735 (\$367-\$5,281) for the Medicaid DMD cohort.

Among the commercial DMD cohort, outpatient resource use was the largest driver of costs (median, \$939), followed by costs of medications (\$333). Inpatient costs were negligible for the majority of patients. Among those treated with corticosteroids, corticosteroid use accounted for $30 \%$ of medication costs. Median (IQR) costs in the DMD cohort increased with age, ranging from $\$ 797(\$ 370-\$ 2,230)$ among boys aged 0-3 years $(n=59)$ to $\$ 2,637(\$ 493-\$ 9,518)$ among those aged 26 years and older $(n=141)$ at baseline.

Among the Medicaid DMD cohort, outpatient visits were also the largest driver of costs (median, \$728) followed by medication use (\$211) and inpatient costs. Among 


\section{TABLE 2 Monthly Resource Use Over Study Period for DMD and Comparison Cohorts, by Type of Insurance Coverage ${ }^{\mathrm{a}}$}

\begin{tabular}{|c|c|c|c|c|c|}
\hline \multirow[b]{3}{*}{ Type of resource } & \multicolumn{2}{|c|}{ Commercial } & \multicolumn{3}{|c|}{ Medicaid } \\
\hline & DMD & Comparison & DMD & \multicolumn{2}{|c|}{ Comparison } \\
\hline & $n=1,420$ & $\mathrm{n}=4,486$ & $n=1,722$ & \multicolumn{2}{|c|}{$n=5,395$} \\
\hline \multicolumn{6}{|l|}{ Medications } \\
\hline Distinct medications dispensed & $2.4 \quad(1.5-4.4)$ & $1.3 \quad(1.0-2.0)$ & $3.5 \quad(1.9-6.6)$ & 1.5 & $(0.0-2.6)$ \\
\hline \multicolumn{6}{|l|}{ Hospitalizations } \\
\hline Any hospitalization, n (\%) & $(25.9)$ & $(3.6)$ & $(31.1)$ & 345 & $(6.4)$ \\
\hline Hospitalizations among those with $\geq 1$ hospitalization & $0.0 \quad(0.0-0.1)$ & $0.0 \quad(0.0-0.1)$ & $0.0 \quad(0.0-0.1)$ & 0.0 & $(0.0-0.1)$ \\
\hline Length of stay per hospitalization (days) & $4.0 \quad(2.0-7.0)$ & $3.0 \quad(2.0-5.0)$ & $4.3 \quad(2.0-8.2)$ & 3.4 & $(2.0-6.0)$ \\
\hline \multicolumn{6}{|l|}{ ED visits } \\
\hline Any ED visit, $n(\%)$ & $(47.8)$ & 1,215 & 1,113 & 2,625 & $(48.7)$ \\
\hline ED visits among those with $\geq 1$ visit & $0.1 \quad(0.0-0.1)$ & $0.0 \quad(0.0-0.1)$ & $0.1 \quad(0.0-0.1)$ & 0.1 & $(0.0-0.1)$ \\
\hline \multicolumn{6}{|l|}{ Outpatient visits } \\
\hline Outpatient visits & $1.4 \quad(0.7-3.1)$ & $0.2 \quad(0.1-0.5)$ & $3.8(1.3-13.9)$ & 0.3 & $(0.1-0.7)$ \\
\hline$\geq 1$ GP visit, n (\%) & $(64.5)$ & 2,074 & 1,179 & 2,670 & $(49.5)$ \\
\hline GP visits among those with $\geq 1$ GP visit & $0.1 \quad(0.0-0.2)$ & $0.0 \quad(0.0-0.1)$ & $0.1 \quad(0.0-0.4)$ & 0.0 & $(0.0-0.1)$ \\
\hline$\geq 1$ specialist visit, $n(\%)$ & 1,378 & 3,509 & $(75.1)$ & 3,441 & $(63.8)$ \\
\hline Specialist visits among those with $\geq 1$ specialist visit & $0.5 \quad(0.3-0.9)$ & $0.1 \quad(0.0-0.3)$ & $0.2 \quad(0.0-0.5)$ & 0.1 & $(0.0-0.2)$ \\
\hline
\end{tabular}

Note: Data reported as median (IQR), unless otherwise stated.

${ }^{a} D M D$ and comparison cohorts with a minimum of 1-year follow-up.

$D M D=$ Duchenne muscular dystrophy; $E D=$ emergency department; $G P=$ general practitioner; $I Q R=$ interquartile range.

those treated with corticosteroids, corticosteroid use accounted for $18 \%$ of medication costs. Median (IQR) costs in the DMD cohort ranged from \$229 (\$45-\$923) among boys aged $0-3$ years $(n=91)$ to $\$ 3,480(\$ 359-\$ 10,754)$ among those aged 26 years and older at baseline $(n=144$; Table 3$)$.

For members of both DMD cohorts, increases in outpatient costs were the largest drivers of differences in costs by age; the contribution of medication costs was relatively consistent across age categories (Figure 2). Costs as observed in the databases were substantially higher for the DMD cohorts than the comparison non-DMD cohorts. Median monthly costs among the commercial DMD cohort were 25.8-fold higher than among the commercial comparison cohort and 64.3-fold higher among the Medicaid DMD cohort vs the Medicaid comparison cohort (Table 3).

\section{SENSITIVITY ANALYSES}

The results of sensitivity analyses that used more specific definitions for DMD did not differ dramatically from the base-case results (Supplementary Table 3). When DMD was defined by additionally requiring at least 1 specialist diagnosis, the median monthly cost per person for the commercial DMD cohort increased from $\$ 1,883$ to $\$ 1,903(P=0.8200)$ and from $\$ 1,735$ to $\$ 1,969$ for the Medicaid $\operatorname{DMD}$ cohort $(\mathrm{P}=0.0060)$. When the DMD definition excluded myoneural disorder and similar diseases, median monthly costs per person decreased from $\$ 1,883$ to $\$ 1,823$ for the commercial DMD cohort $(P=0.5027)$ and from $\$ 1,735$ to $\$ 1,665$ for the Medicaid DMD cohort $(P=0.4783)$.

When the analyses of resource use were restricted to data from the first year of follow-up per patient, the percentages having at least 1 hospitalization, visit to the general practitioner, or specialists were lower in both DMD cohorts. While the rates of general practitioner visits and hospitalizations remained higher among the Medicaid DMD cohort than the commercial DMD cohort, the percentage with at least 1 specialist visit remained higher among the commercial DMD cohort (Supplementary Table 4).

The amount of missing costs data was higher in the Medicaid DMD cohort than the commercial DMD cohort. As an example, none of the commercial DMD cohort patients were missing costs for all of their health insurance 


\section{FIGURE 2 Median Total Monthly Costs in Commercial and Medicaid DMD Cohorts}

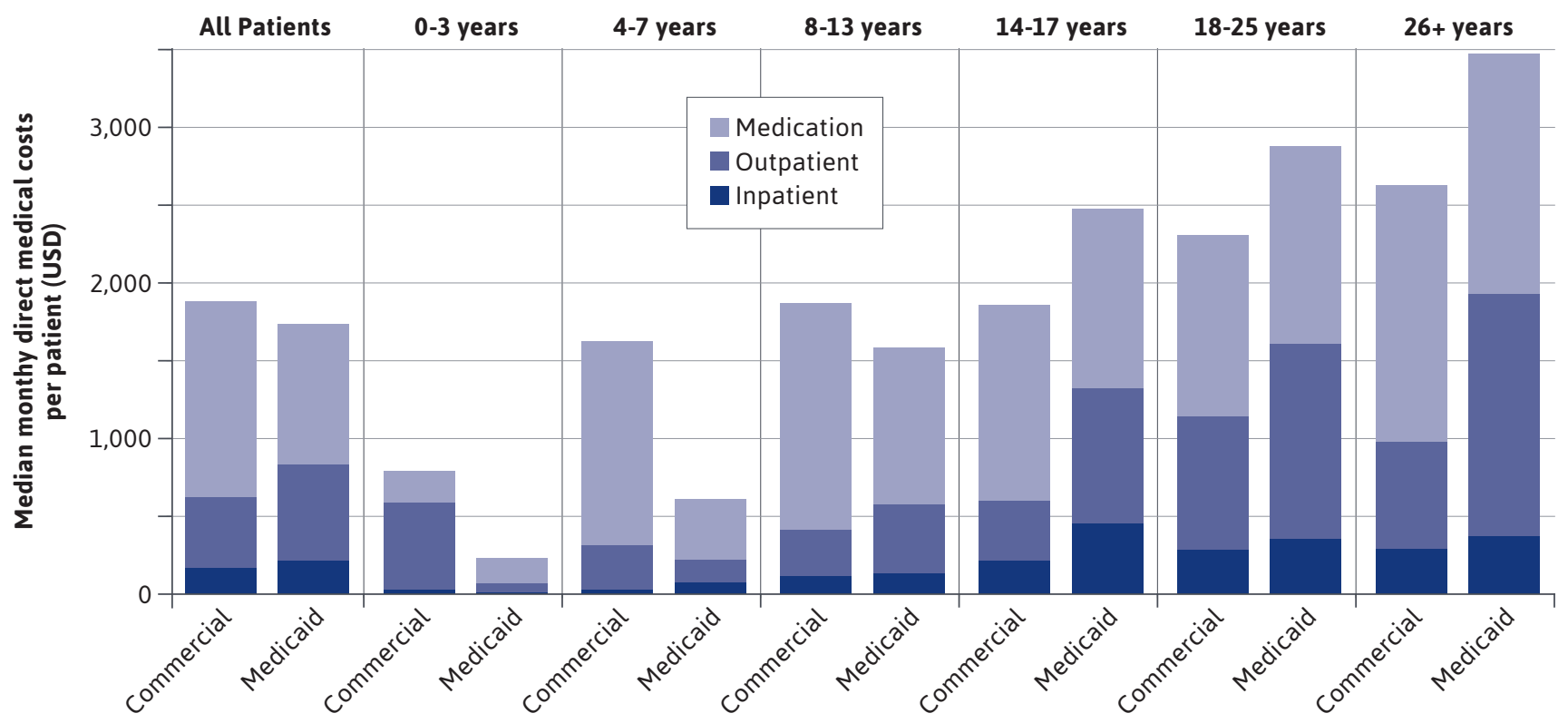

Note: Costs are presented in 2018 US dollars.

DMD = Duchenne muscular dystrophy.

claims, whereas this occurred for 67 (3.3\%) members of the Medicaid DMD cohort. When missing costs for all encounters with \$0 charges assigned were imputed, median (IQR) monthly costs increased to $\$ 2,018$ (\$696-\$7,053; $\mathrm{P}=0.4579$ for comparison to base-case results) in the commercial DMD cohort and to $\$ 2,071(\$ 663-\$ 5,801 ; \mathrm{P}<0.0001)$ in the Medicaid DMD cohort.

\section{Discussion}

Few claims studies have yet characterized real-world populations of DMD, their treatment, and their attendant cost of care in the United States, particularly considering patients with different types of health insurance. The largest study addressing these objectives to date used data from commercially insured individuals and was conducted before the release of guidelines recommending the use of corticosteroids as standard of care, ${ }^{10,22}$ which has resulted in a shift in the clinical course, pattern, and timing of complications in DMD. ${ }^{12}$ Contemporary estimates of the demographics, comorbidity burden, and costs of care among patients with DMD on either commercial and Medicaid plans are presently lacking.
This real-world study used data from the large representative MarketScan databases to identify almost 2,000 patients with DMD covered under commercial insurance plans and a similar number of patients covered under Medicaid insurance. DMD patients with commercial and Medicaid coverage were similar in many respects, including in their median age and age distribution. The comorbidity burden in both DMD cohorts was high-higher among those in the Medicaid DMD cohort than in the commercial DMD cohort and higher in both the DMD cohorts than in their respective non-DMD comparison cohorts.

Monthly resource use was higher for patients with DMD than for patients without DMD and higher among members of the Medicaid DMD cohort than the commercial DMD cohort, including when stratified by age or when the observation period was fixed to a 1-year duration postindex in a sensitivity analysis. Resource use was higher among the Medicaid DMD cohort, especially for outpatient visits, possibly because of differences in comorbidity burden, despite similarities in age distributions between the 2 DMD cohorts. In addition, there could have been innate differences between the coding and billing practices in Medicaid and commercial databases, as well as any local regional differences in the states included in each database 


\section{TABLE 3 Monthly Costs Among Commercial and Medicaid DMD and Comparison Cohorts, by Age Group}

\begin{tabular}{|c|c|c|c|c|c|c|c|c|c|c|c|c|c|c|}
\hline & \multirow{2}{*}{\multicolumn{2}{|c|}{ All patients }} & \multicolumn{12}{|c|}{ Baseline age group } \\
\hline & & & \multicolumn{2}{|c|}{$0-3$ years } & \multicolumn{2}{|c|}{ 4-7 years } & \multicolumn{2}{|c|}{ 8-13 years } & \multicolumn{2}{|c|}{$14-17$ years } & \multicolumn{2}{|c|}{$18-25$ years } & \multicolumn{2}{|c|}{$26+$ years } \\
\hline & 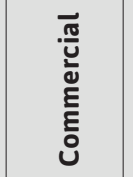 & 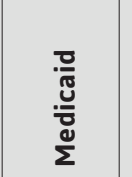 & 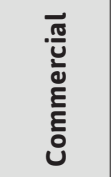 & $\frac{.0}{\pi}$ & $\begin{array}{l}\frac{\pi}{U} \\
\frac{\pi}{2} \\
\frac{E}{E} \\
0 \\
0\end{array}$ & . 쥮 & 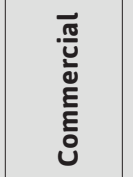 & $\begin{array}{l}. \frac{\pi}{\pi} \\
\frac{0}{\pi} \\
\frac{0}{\Sigma}\end{array}$ & 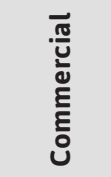 &  & $\begin{array}{l}\frac{\pi}{U} \\
\frac{\pi}{2} \\
\frac{\Xi}{E} \\
0\end{array}$ & $\begin{array}{l}\frac{.0}{\pi} \\
\frac{0}{0} \\
\frac{0}{\Sigma}\end{array}$ & 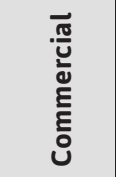 & . \\
\hline \multicolumn{15}{|l|}{ DMD cohorts } \\
\hline & $n=1,420$ & $n=1,722$ & $n=59$ & $\mathrm{n}=91$ & $n=184$ & $n=235$ & $n=378$ & $n=468$ & $n=259$ & $n=326$ & $n=399$ & $n=458$ & $\mathrm{n}=141$ & $n=144$ \\
\hline Medications & $\begin{array}{c}\$ 333 \\
(\$ 38- \\
\$ 2,437)\end{array}$ & \begin{tabular}{|c|}
$\$ 211$ \\
$(\$ 12-$ \\
$\$ 1,533)$
\end{tabular} & $\begin{array}{l}\$ 58 \\
(\$ 6- \\
\$ 386)\end{array}$ & $\begin{array}{c}\$ 12 \\
(\$ 0 \\
-\$ 246)\end{array}$ & $\begin{array}{c}\$ 268 \\
(\$ 47- \\
\$ 2,623)\end{array}$ & $\begin{array}{c}\$ 92 \\
(\$ 0- \\
\$ 658)\end{array}$ & \begin{tabular}{|c|}
$\$ 282$ \\
$(\$ 26-$ \\
$\$ 3,480)$
\end{tabular} & \begin{tabular}{|c|}
$\$ 184$ \\
$(\$ 21-$ \\
$\$ 1,580)$
\end{tabular} & $\begin{array}{c}\$ 372 \\
(\$ 78- \\
\$ 1,729)\end{array}$ & \begin{tabular}{|c|}
$\$ 353$ \\
$(\$ 22-$ \\
$\$ 2,430)$
\end{tabular} & $\begin{array}{c}\$ 444 \\
(\$ 40- \\
\$ 2,310)\end{array}$ & \begin{tabular}{|c|}
$\$ 330$ \\
$(\$ 28-$ \\
$\$ 1,748)$
\end{tabular} & $\begin{array}{c}\$ 730 \\
(\$ 72- \\
\$ 4,539)\end{array}$ & $\begin{array}{c}\$ 325 \\
(\$ 4- \\
\$ 2,048)\end{array}$ \\
\hline Inpatient & $\begin{array}{c}\$ 0 \\
(\$ 0- \\
\$ 117)\end{array}$ & $\begin{array}{l}\$ 0 \\
(\$ 0- \\
\$ 93)\end{array}$ & $\begin{array}{c}\$ 0 \\
(\$ 0-\$ 0)\end{array}$ & $\begin{array}{c}\$ 0 \\
(\$ 0-\$ 0)\end{array}$ & $\begin{array}{c}\$ 0 \\
(\$ 0-\$ 0)\end{array}$ & $\begin{array}{c}\$ 0 \\
(\$ 0-\$ 0)\end{array}$ & $\begin{array}{c}\$ 0 \\
(\$ 0-\$ 0)\end{array}$ & $\begin{array}{c}\$ 0 \\
(\$ 0-\$ 0)\end{array}$ & $\begin{array}{c}\$ 0 \\
(\$ 0- \\
\$ 478)\end{array}$ & $\begin{array}{c}\$ 0 \\
(\$ 0- \\
\$ 270)\end{array}$ & $\begin{array}{c}\$ 0 \\
(\$ 0- \\
\$ 392)\end{array}$ & $\begin{array}{c}\$ 0 \\
(\$ 0- \\
\$ 352)\end{array}$ & $\begin{array}{c}\$ 0 \\
(\$ 0- \\
\$ 401)\end{array}$ & $\begin{array}{c}\$ 0 \\
(\$ 0- \\
\$ 585)\end{array}$ \\
\hline Outpatient & $\begin{array}{c}\$ 939 \\
(\$ 382- \\
\$ 1,948)\end{array}$ & $\begin{array}{c}\$ 728 \\
(\$ 192- \\
\$ 2,301)\end{array}$ & $\begin{array}{c}\$ 642 \\
(\$ 281- \\
\$ 1,532)\end{array}$ & $\begin{array}{l}\$ 208 \\
(\$ 36- \\
\$ 467)\end{array}$ & $\begin{array}{c}\$ 977 \\
(\$ 487- \\
\$ 1,715)\end{array}$ & $\begin{array}{l}\$ 354 \\
(\$ 97- \\
\$ 797)\end{array}$ & $\begin{array}{c}\$ 923 \\
(\$ 456- \\
\$ 1,767)\end{array}$ & $\begin{array}{c}\$ 652 \\
(\$ 245- \\
\$ 1,606)\end{array}$ & $\begin{array}{c}\$ 1,116 \\
(\$ 451- \\
\$ 1,801)\end{array}$ & $\begin{array}{c}\$ 961 \\
(\$ 267- \\
\$ 2,581)\end{array}$ & $\begin{array}{c}\$ 947 \\
(\$ 297- \\
\$ 2,275)\end{array}$ & \begin{tabular}{|c|}
$\$ 1,447$ \\
$(\$ 284-$ \\
$\$ 3,426)$
\end{tabular} & $\begin{array}{c}\$ 795 \\
(\$ 208- \\
\$ 2,605)\end{array}$ & $\begin{array}{l}\$ 1,571 \\
(\$ 150- \\
\$ 5,413)\end{array}$ \\
\hline Total & $\begin{array}{c}\$ 1,883 \\
(\$ 657- \\
\$ 6,796)\end{array}$ & $\begin{array}{c}\$ 1,735 \\
(\$ 367- \\
\$ 5,281)\end{array}$ & $\begin{array}{c}\$ 797 \\
(\$ 370- \\
\$ 2,230)\end{array}$ & $\begin{array}{l}\$ 229 \\
(\$ 45- \\
\$ 923)\end{array}$ & $\begin{array}{c}\$ 1,629 \\
(\$ 698- \\
\$ 5,189)\end{array}$ & $\begin{array}{c}\$ 609 \\
(\$ 138- \\
\$ 1,740)\end{array}$ & \begin{tabular}{|l|}
$\$ 1,864$ \\
$(\$ 685-$ \\
$\$ 7,070)$
\end{tabular} & \begin{tabular}{|c|}
$\$ 1,588$ \\
$(\$ 448-$ \\
$\$ 4,468)$
\end{tabular} & $\begin{array}{l}\$ 1,856 \\
(\$ 732- \\
\$ 6,243)\end{array}$ & \begin{tabular}{|l|}
$\$ 2,476$ \\
$(\$ 636-$ \\
$\$ 7,063)$
\end{tabular} & $\begin{array}{c}\$ 2,313 \\
(\$ 592- \\
\$ 7,534)\end{array}$ & \begin{tabular}{|l|}
$\$ 2,882$ \\
$(\$ 733-$ \\
$\$ 7,269)$
\end{tabular} & $\begin{array}{l}\$ 2,637 \\
(\$ 493- \\
\$ 9,518)\end{array}$ & $\begin{array}{c}\$ 3,480 \\
(\$ 359- \\
\$ 10,754)\end{array}$ \\
\hline \multicolumn{15}{|c|}{ Comparison cohorts } \\
\hline & $n=4,486$ & $n=5,395$ & $\mathrm{n}=190$ & $\mathrm{n}=262$ & $\mathrm{n}=579$ & $\mathrm{n}=817$ & $n=1,246$ & $n=1,639$ & $n=971$ & $n=1,128$ & $n=1,135$ & $n=1,149$ & $n=365$ & $\mathrm{n}=400$ \\
\hline Medications & $\begin{array}{l}\$ 6 \\
(\$ 0- \\
\$ 85)\end{array}$ & $\begin{array}{l}\$ 0 \\
(\$ 0- \\
\$ 33)\end{array}$ & $\begin{array}{l}\$ 12 \\
(\$ 1- \\
\$ 76)\end{array}$ & $\begin{array}{l}\$ 0 \\
(\$ 0- \\
\$ 37)\end{array}$ & $\begin{array}{l}\$ 11 \\
(\$ 0- \\
\$ 79)\end{array}$ & $\begin{array}{l}\$ 1 \\
(\$ 0- \\
\$ 58)\end{array}$ & $\begin{array}{l}\$ 10 \\
(\$ 0- \\
\$ 130)\end{array}$ & $\begin{array}{l}\$ 0 \\
(\$ 0- \\
\$ 46)\end{array}$ & $\begin{array}{c}\$ 7 \\
(\$ 0- \\
\$ 131)\end{array}$ & $\begin{array}{l}\$ 0 \\
(\$ 0- \\
\$ 22)\end{array}$ & $\begin{array}{l}\$ 2 \\
(\$ 0- \\
\$ 41)\end{array}$ & $\begin{array}{l}\$ 0 \\
(\$ 0- \\
\$ 13)\end{array}$ & $\begin{array}{l}\$ 6 \\
(\$ 0- \\
\$ 91)\end{array}$ & $\begin{array}{l}\$ 0 \\
(\$ 0- \\
\$ 36)\end{array}$ \\
\hline Inpatient & $\begin{array}{c}\$ 0 \\
(\$ 0-\$ 0)\end{array}$ & $\begin{array}{c}\$ 0 \\
(\$ 0-\$ 0)\end{array}$ & $\begin{array}{c}\$ 0 \\
(\$ 0-\$ 0)\end{array}$ & $\begin{array}{c}\$ 0 \\
(\$ 0-\$ 0)\end{array}$ & $\begin{array}{c}\$ 0 \\
(\$ 0-\$ 0)\end{array}$ & $\begin{array}{c}\$ 0 \\
(\$ 0-\$ 0)\end{array}$ & $\begin{array}{c}\$ 0 \\
(\$ 0-\$ 0)\end{array}$ & $\begin{array}{c}\$ 0 \\
(\$ 0-\$ 0)\end{array}$ & $\begin{array}{c}\$ 0 \\
(\$ 0-\$ 0)\end{array}$ & $\begin{array}{c}\$ 0 \\
(\$ 0-\$ 0)\end{array}$ & $\begin{array}{c}\$ 0 \\
(\$ 0-\$ 0)\end{array}$ & $\begin{array}{c}\$ 0 \\
(\$ 0-\$ 0)\end{array}$ & $\begin{array}{c}\$ 0 \\
(\$ 0-\$ 0)\end{array}$ & $\begin{array}{c}\$ 0 \\
(\$ 0-\$ 0)\end{array}$ \\
\hline Outpatient & $\begin{array}{l}\$ 45 \\
(\$ 16- \\
\$ 121)\end{array}$ & $\begin{array}{l}\$ 19 \\
(\$ 0- \\
\$ 75)\end{array}$ & $\begin{array}{l}\$ 76 \\
(\$ 43- \\
\$ 174) \\
\end{array}$ & $\begin{array}{l}\$ 23 \\
(\$ 0- \\
\$ 77)\end{array}$ & $\begin{array}{l}\$ 42 \\
(\$ 20- \\
\$ 109)\end{array}$ & $\begin{array}{l}\$ 32 \\
(\$ 0- \\
\$ 82)\end{array}$ & $\begin{array}{l}\$ 51 \\
(\$ 25- \\
\$ 124) \\
\end{array}$ & $\begin{array}{l}\$ 22 \\
(\$ 0- \\
\$ 71)\end{array}$ & $\begin{array}{l}\$ 46 \\
(\$ 16- \\
\$ 132) \\
\end{array}$ & $\begin{array}{l}\$ 16 \\
(\$ 0- \\
\$ 66)\end{array}$ & $\begin{array}{c}\$ 30 \\
(\$ 5- \\
\$ 100) \\
\end{array}$ & $\begin{array}{l}\$ 9 \\
\$ 0- \\
\$ 75)\end{array}$ & $\begin{array}{c}\$ 32 \\
(\$ 11- \\
\$ 134) \\
\end{array}$ & $\begin{array}{l}\$ 13 \\
(\$ 0- \\
\$ 119) \\
\end{array}$ \\
\hline Total & $\begin{array}{l}\$ 73 \\
(\$ 21- \\
\$ 308)\end{array}$ & $\begin{array}{l}\$ 27 \\
(\$ 0- \\
\$ 161)\end{array}$ & $\begin{array}{l}\$ 121 \\
(\$ 52- \\
\$ 261)\end{array}$ & $\begin{array}{c}\$ 30 \\
(\$ 0- \\
\$ 162)\end{array}$ & $\begin{array}{l}\$ 73 \\
(\$ 27- \\
\$ 245)\end{array}$ & $\begin{array}{l}\$ 46 \\
(\$ 0- \\
\$ 178)\end{array}$ & $\begin{array}{l}\$ 86 \\
(\$ 32- \\
\$ 348) \\
\end{array}$ & $\begin{array}{l}\$ 34 \\
(\$ 0- \\
\$ 177)\end{array}$ & $\begin{array}{l}\$ 81 \\
(\$ 23- \\
\$ 409)\end{array}$ & $\begin{array}{c}\$ 23 \\
(\$ 0- \\
\$ 134)\end{array}$ & $\begin{array}{c}\$ 49 \\
(\$ 7- \\
\$ 219)\end{array}$ & $\begin{array}{c}\$ 12 \\
(\$ 0- \\
\$ 151)\end{array}$ & $\begin{array}{l}\$ 63 \\
(\$ 14- \\
\$ 358) \\
\end{array}$ & $\begin{array}{l}\$ 17 \\
(\$ 0- \\
\$ 261)\end{array}$ \\
\hline
\end{tabular}

Notes: Data reported as median (IQR), unless otherwise stated. Costs are reported in 2018 US dollars.

that could have contributed to these observed differences in resource use. ${ }^{37}$ Given the uncertainty with exactly how payments occur for patients in different plans and whether these are truly costs or charges, the resource use estimates may be a better indicator of economic burden, with a view to generalizability. Finally, observed differences between the DMD cohorts could also be partly explained by factors such as socioeconomic status that could also have affected comorbidity burden or transition of patients through different types of insurance plans as DMD progresses, ${ }^{38,39}$ the impact of which would need to be confirmed in a formal comparative assessment using data that document more of these potentially important covariates.
Despite the higher resource use and comorbidities among members of the Medicaid DMD cohort, median direct medical costs per patient were similar between the DMD cohorts, at approximately $\$ 1,800$ per month, which corresponded to an estimated median annual cost of approximately $\$ 22,000$ per patient in either DMD cohort. Costs were also similar between the DMD cohorts when considered according to age at cohort entry. Direct comparison with other studies is challenging because other studies of the economic burden of DMD presented estimates of mean costs per month, which can be heavily influenced by outliers, particularly in smaller samples. ${ }^{40}$ Therefore, it is not surprising that the cost estimates from those studies were slightly higher. 
The previous claims study of 75 commercially insured patients estimated a mean cost of $\$ 23,005$ per patient per year (in 2010 USD), ${ }^{22}$ and an international, cross-sectional, survey-based study that included 284 US patients estimated a mean of $\$ 22,500$ per patient per year (in 2012 international dollars) based on self-reported resource use and not stratified according to payer type. ${ }^{11}$

It is not clear whether the differences in costs observed between commercial and Medicaid are attributable to different payment schedules or capitated costs; these details were not available in the datasets. It is also notable that, when stratified by age, older members of the Medicaid DMD cohort had higher monthly cost estimates compared with commercial DMD patients in the same age category, although the opposite was true for younger DMD patients. Overall, costs were higher among older DMD patients.

A number of methodological points warrant further discussion to help guide the interpretation of the results of this study. First, the generally short follow-up (2-4 years) and differences in median follow-up duration between the cohorts makes directly comparing some outcomes between the commercial and Medicaid DMD cohorts challenging. For example, while corticosteroids are known to delay progression of DMD, ${ }^{21,41-44}$ expected levels of corticosteroid use were not observed in this study, presumably because not all cohort members appeared in the data during the critical window in which these would have been prescribed. Thus, a comprehensive understanding of each individual's lifetime corticosteroid use was not possible and may impact the interpretation of comparisons between the DMD cohorts. It should be noted that the low rate of observed corticosteroid use in this study is consistent with other reports from studies using real-world data and highlights the need for additional treatment options for DMD.43

Identifying patients based on DMD-genotype specific treatments (such as eteplirsen) or ICD-10-CM diagnositic codes was also explored but did not identify any additional patients beyond those already captured by the study inclusion criteria. The number of patients identified with eteplirsen use was low, since this treatment had only become available for the subset of eligible DMD patients towards the end of the study period.$^{45}$ Finally, all-cause costs (rather than DMD-specific costs) were presented, since it was assumed that in this young but severely affected patient population, most observed health care resource use would be targeted towards managing complications of DMD.

A key strength of these analyses was the use of wellvalidated datasets that provided a large sample size of DMD patients, including children and young adults, from commercial and Medicaid insurance plans. Restricting the economic analyses to cohorts with at least 1 year of follow-up data allowed a more robust estimate of health care resource use by reducing the impact of any potential individuals with short follow-up but high costs on the estimates. Results of sensitivity analyses that used different definitions of DMD were very consistent with those of the base-case analyses, providing support for the robustness of the overall study findings.

\section{LIMITATIONS}

This study does have some limitations to consider. As with any retrospective study, findings are limited by data availability and the duration of follow-up within the datasets, which was relatively short. The period of data availability for the Medicaid dataset was slightly longer (an additional 6 months) than for the commercial dataset. To ensure the largest sample size and longest follow-up, the duration of data availability was retained as provided by the data holders. Nonetheless, a person-time approach was used for the data analysis such that any differences in the exact period of data availability across datasets would not bias the findings of the analysis.

The comparability of the Medicaid and commercial cohorts were limited by numerous factors, including that the eligible study pools were different (18.7 million in commercial vs 6.0 million in Medicaid males aged 30 years or under), as well as likely differences in unmeasured factors (eg, socioeconomic status, transitions between plans, differences in access, and geography) between the groups that could also influence health care resource use and costs. The availability of data on out-of-pocket costs in the datasets was limited and varied between the commercial and Medicaid datasets, and other potential elements of the economic burden, such as family or societal costs, were not reflected in these datasets.

It is also important to note that reasons for attrition were not specified in the datasets, so it was unclear if individuals were lost to follow-up due to changing insurance plans, moving, or mortality. Furthermore, it was unknown if the distribution of reasons why patients were lost to follow-up were similar in the commercial and Medicaid cohorts.

In addition, because claims data are collected for the purposes of billing and not for research, errors in coding may affect the estimates calculated. Also, claims datasets do not contain the laboratory results required to confirm a diagnosis of DMD, and there is no unique ICD diagnostic code to identify DMD patients. To reduce the likelihood of misclassification of exposure, previously validated definitions for DMD were adapted for use in the current analysis and were tested in sensitivity analyses..$^{22,46,47}$ While little variability in estimates was observed based on the 
sensitivity analyses, without comparison to a gold standard (eg, cases identified from a chart review), the optimal DMD case definition for use in administrative data analyses could not be confirmed.

Finally, the results of this study are specific to individuals covered under a subset of commercial and Medicaid insurance plans and may not be generalizable to all commercial and Medicaid payer segments in the United States.

\section{Conclusions}

In this large real-world study of patients with DMD, the distribution of demographic characteristics was comparable between the commercial and Medicaid DMD cohorts, and monthly direct medical costs were similar, although patients with Medicaid coverage had higher resource use. This study provides contemporary data on the characteristics of DMD patients, their health care resource use, and attendant economic burden to the payer.

\section{DISCLOSURES}

This study was funded by Sarepta Therapeutics, Inc. Klimchak and Gooch are employees of Sarepta Therapeutics Inc. Szabo, Qian, and Popoff are employees of Broadstreet HEOR, which received funds from Sarepta Therapeutics, Inc., for work on this study. Iannaccone has received research funding or consulting fees from Avexis, Biogen, Fibrogen, Mallinkrodt, Regeneron, Sarepta Therapeutics, Inc., Scholar Rock, PTC Therapeutics, Pfizer, MDA, CureSMA, NIH, Genentech-Roche, and BCBS. Publication of the study results was not contingent on the sponsor's approval or censorship of the manuscript.

Information from this study was presented, in part, at the AMCP Virtual Annual Meeting, April 21-24, 2020.

\section{REFERENCES}

1. Wein N, Alfano L, Flanigan KM. Genetics and emerging treatments for Duchenne and Becker muscular dystrophy. Pediatr Clin North Am. 2015;62(3):723-42.

2. Crisafulli S, Sultana J, Fontana A, Salvo F, Messina S, Trifiro G. Global epidemiology of Duchenne muscular dystrophy: an updated systematic review and meta-analysis. Orphanet J Rare Dis. 2020;15(1):141.

3. Yiu EM, Kornberg AJ. Duchenne muscular dystrophy. J Paediatr Child Health. 2015;51(8):759-64.

4. Mirski KT, Crawford TO. Motor and cognitive delay in Duchenne muscular dystrophy: implication for early diagnosis. J Pediatr. 2014;165(5):1008-10.

5. Connolly AM, Florence JM, Cradock MM, et al. One year outcome of boys with Duchenne muscular dystrophy using the Bayley-III scales of infant and toddler development. Pediatr Neurol. 2014;50(6):557-63.

6. Connolly AM, Florence JM, Cradock MM, et al. Motor and cognitive assessment of infants and young boys with Duchenne muscular dystrophy: results from the Muscular Dystrophy Association DMD Clinical Research Network. Neuromuscul Disord. 2013;23(7):529-39.

7. McDonald CM, Henricson EK, Abresch RT, et al. The cooperative international neuromuscular research group Duchenne natural history study-a longitudinal investigation in the era of glucocorticoid therapy: design of protocol and the methods used. Muscle Nerve. 2013;48(1):32-54.

8. Parent Project Muscular Dystrophy. The Duchenne Registry. 2019. Accessed April 30, 2021. https://www.duchenneregistry. org/

9. Centers for Disease Control and Prevention. Muscular dystrophy research and tracking. 2019. Accessed April 30, 2021. https://www.cdc.gov/ncbddd/musculardystrophy/research.html
10. Birnkrant DJ, Bushby K, Bann CM, et al. Diagnosis and management of Duchenne muscular dystrophy, part 1: diagnosis, and neuromuscular, rehabilitation, endocrine, and gastrointestinal and nutritional management. Lancet Neurol. 2018;17(3):251-67.

11. Landfeldt E, Lindgren P, Bell CF, et al. The burden of Duchenne muscular dystrophy: an international, cross-sectional study. Neurology. 2014;83(6):529-536.

12. Ryder S, Leadley RM, Armstrong N, et al. The burden, epidemiology, costs and treatment for Duchenne muscular dystrophy: an evidence review. Orphanet J Rare Dis. 2017;12(1):79.

13. Andrews JG, Wahl RA. Duchenne and Becker muscular dystrophy in adolescents: current perspectives. Adolesc Health Med Ther. 2018;9:53-63.

14. Hendriksen RGF, Vles JSH, Aalbers MW, Chin RFM, Hendriksen JGM. Brainrelated comorbidities in boys and men with Duchenne muscular dystrophy: a descriptive study. Eur J Paediatr Neurol. 2018;22(3):488-97.

15. Ricotti V, Mandy WP, Scoto M, et al. Neurodevelopmental, emotional, and behavioural problems in Duchenne muscular dystrophy in relation to underlying dystrophin gene mutations. Dev Med Child Neurol. 2016;58(1):77-84.

16. Snow WM, Anderson JE, Jakobson LS. Neuropsychological and neurobehavioral functioning in Duchenne muscular dystrophy: a review. Neurosci Biobehav Rev. 2013;37(5):743-52.

17. Hendriksen JG, Vles JS. Neuropsychiatric disorders in males with duchenne muscular dystrophy: frequency rate of attention-deficit hyperactivity disorder (ADHD), autism spectrum disorder, and obsessive-compulsive disorder. J Child Neurolol. 2008;23(5):477-81.

18. Pane M, Messina S, Bruno C, et al. Duchenne muscular dystrophy and epilepsy. Neuromuscul Disord. 2013;23(4):313-15.

19. Stromberg A, Darin N, Kroksmark AK, Tulinius M. S.P.31 What was the age and cause of death in patients with Duchenne muscular dystrophy in Sweden during 2000-2010? Neuromuscul Disord. 2012;22(9):880-81. 
20. Bello L, Morgenroth LP, GordishDressman $\mathrm{H}$, et al. DMD genotypes and loss of ambulation in the CINRG Duchenne Natural History Study. Neurology. 2016;87(4):401-09.

21. McDonald CM, Henricson EK, Abresch RT, et al. Long-term effects of glucocorticoids on function, quality of life, and survival in patients with Duchenne muscular dystrophy: a prospective cohort study. Lancet. 2018;391(10119):451-61.

22. Thayer S, Bell C, McDonald CM. The direct cost of managing a rare disease: assessing medical and pharmacy costs associated with duchenne muscular dystrophy in the United States. J Manag Care Spec Pharm. 2017;23(6):633-41. doi: 10.18553/jmcp.2017.23.6.633

23. Bello L, D'Angelo G, Villa M, et al. Genetic modifiers of respiratory function in Duchenne muscular dystrophy. Ann Clin Transl Neurol. 2020;7(5):786-98.

24. IBM Watson Health. Multi-State Medicaid Database. 2017. Accessed August 26, 2021. https://www.ibm.com/products/marketscan-research-databases/ databases

25. IBM Watson Health. Commercial Claims and Encounters Database and Medicare Supplemental and Coordination of Benefits Database. 2017. Accessed August 26, 2021. https://www.ibm.com/products/ marketscan-research-databases/ databases

26. Gauthier G, Guérin A, Zhdanava M, et al. Treatment patterns, healthcare resource utilization, and costs following first-line antidepressant treatment in major depressive disorder: a retrospective US claims database analysis. BMC Psychiatry. 2017;17(1):222.

27. Marsico M, Mehta V, Chastek B, Liaw K-L, Derkay C. Estimating the incidence and prevalence of juvenile-onset recurrent respiratory papillomatosis in publicly and privately insured claims databases in the United States. Sex Transm Dis. 2014;41(5):300-05.
28. Szabo SM, Gooch K, Schermer C, et al. Association between cumulative anticholinergic burden and falls and fractures in patients with overactive bladder: US-based retrospective cohort study. BMJ Open. 2019;9(5):e026391.

29. Wu JM, Matthews CA, Conover MM, Pate V, Funk MJ. Lifetime risk of stress incontinence or pelvic organ prolapse surgery. Obstet Gynecol. 2014;123(6):1201-06

30. Zhao Y, Johnston SS, Smith DM, McMorrow D, Krege J, Krohn K. Association between teriparatide adherence and healthcare utilization and costs among hip fracture patients in the United States. Bone. 2014;60:221-26.

31. Broulette J, Yu H, Pyenson B, Iwasaki K, Sato R. The incidence rate and economic burden of community-acquired pneumonia in a working-age population. Am Health Drug Benefits. 2013;6(8):494.

32. Lykins J, Wang K, Wheeler K, et al. Understanding toxoplasmosis in the United States through "large data" analyses. Clin Infect Dis. 2016;63(4):468-75.

33. Song X, Shi N, Badamgarav E, et al. Cost burden of second fracture in the US health system. Bone. 2011;48(4):828-36.

34. Durden E, Walker D, Gray SL, Fowler R, Juneau P, Gooch KG. The direct and indirect costs due to work loss associated with overactive bladder in the United States. Paper presented at: American Urological Association; May 12-16, 2017; Boston, MA.

35. Quan H, Sundararajan V, Halfon P, et al. Coding algorithms for defining comorbidities in ICD-9-CM and ICD-10 administrative data. Med Care. 2005;43(11):1130-39.

36. US Bureau of Labor Statistics. Consumer Price Index. 2019. Accessed August 31, 2021. https://www.bls.gov/cpi/ research-series/r-cpi-u-rs-home.htm.

37. Alguire PC. Undersanding capitation. 2021. Accessed January 8, 2021. https://www.acponline.org/about-acp/ about-internal-medicine/career-paths/ residency-career-counseling/guidance/ understanding-capitation
38. Archer D, Mamor T. Medicare and commercial health insurance: the fundamental difference. Health Affairs Blog. February 15, 2012. Accessed August 26, 2021. https://www.healthaffairs.org/ do/10.1377/hblog20120215.016980/full/

39. Lindley LC, Lyon ME. A profile of children with complex chronic conditions at end of life among Medicaid beneficiaries: implications for health care reform. J Palliat Med. 2013;16(11):1388-93.

40. Mani K, Lundkvist J, Holmberg L, Wanhainen A. Challenges in analysis and interpretation of cost data in vascular surgery. J Vasc Surg. 2010;51(1):148-54.

41. Barber BJ, Andrews JG, Lu Z, et al. Oral corticosteroids and onset of cardiomyopathy in Duchenne muscular dystrophy. J Pediatr. 2013;163(4):1080-84.e1081.

42. Bello L, Gordish-Dressman H, Morgenroth LP, et al. Prednisone/prednisolone and deflazacort regimens in the CINRG Duchenne Natural History Study. Neurology. 2015;85(12):1048-55.

43. Cowen L, Mancini M, Martin A, Lucas A, Donovan JM. Variability and trends in corticosteroid use by male United States participants with Duchenne muscular dystrophy in the Duchenne Registry. BMC Neurol. 2019;19(1):84.

44. Kim S, Zhu Y, Romitti PA, et al. Associations between timing of corticosteroid treatment initiation and clinical outcomes in Duchenne muscular dystrophy. Neuromuscul Disord. 2017;27(8):730-37.

45. Aartsma-Rus A, Krieg AM. FDA approves eteplirsen for Duchenne muscular dystrophy: the next chapter in the eteplirsen saga. Nucleic Acid Ther. 2017;27(1):1-3.

46. Smith MG, Royer J, Mann JR, McDermott S. Using administrative data to ascertain true cases of muscular dystrophy: rare disease surveillance. JMIR Public Health Surveill. 2017;3(1):e2.

47. Larkindale J, Yang W, Hogan PF, et al. Cost of illness for neuromuscular diseases in the United States. Muscle Nerve. 2014;49(3):431-38. 